\title{
Preclinical development of Ramizol, an antibiotic belonging to a new class, for the treatment of Clostridium difficile colitis
}

\author{
Shasha Rao ${ }^{1}$, Clive A Prestidge ${ }^{1}$, Lynn Miesel $^{2}$, Deb Sweeney ${ }^{3}$, Dean L Shinabarger ${ }^{3}$ and Ramiz A Boulos ${ }^{4,5}$ \\ Antibiotic-resistant bacteria is a major threat to human health and is predicted to become the leading cause of death from \\ disease by 2050 . Despite the recent resurgence of research and development in the area, few antibiotics have reached the \\ market, with most of the recently approved antibiotics corresponding to new uses for old antibiotics, or structurally similar \\ derivatives thereof. We have recently reported an in silico approach that led to the design of an entirely new class of antibiotics \\ for the bacteria-specific mechanosensitive ion channel of large conductance: MscL. Here, we present the preclinical \\ development of one such antibiotic, Ramizol, a first generation antibiotic belonging to that class. We present the lack of \\ interaction between Ramizol and other mammalian channels adding credibility to its MscL selectivity. We determine the \\ pharmacokinetic profile in a rat model and show $<0.1 \%$ of Ramizol is absorbed systemically. We show this non-systemic nature \\ of the antibiotic translates to over $70 \%$ survival of hamsters in a Clostridium difficile colitis model. Lastly, initial in vitro data \\ indicate that resistance to Ramizol occurs at a low frequency. In conclusion, we establish the potential of Ramizol as an \\ effective new treatment for $C$. difficile associated disease.
}

The Journal of Antibiotics (2016) 69, 879-884; doi:10.1038/ja.2016.45; published online 18 May 2016

\section{INTRODUCTION}

Antibiotic-resistant bacteria are a global threat to human health predicted to become the leading cause of death from disease by 2050. The burden from multi-drug-resistant bacteria in the United States alone, costs between USD 17 billion and USD 26 billion annually. ${ }^{1}$ In Australia, it is estimated that such infections claim the lives of over 3000 people each year. ${ }^{2}$ Nonetheless, the antibiotic pipeline has remained deficient and, despite the recent investment in novel antibiotic drug development, few antibiotics have been discovered. ${ }^{2}$ This is at a time when the last line of defence antibiotics such as the vancomycin and, more recently, daptomycin and linezolid, have lost their efficacy against some drug resistant bacteria. The growing frequency of 'superbug' outbreaks has prompted significant research into the development of new antibiotics with novel targets.

Clostridium difficile infections are among some of the most challenging to treat. In the United States, C. difficile is responsible for more than 250000 hospitalizations annually costing more than USD 4 billion in health-care expenses. It is estimated that as many as 15000 lives succumb to these infections each year in the United States alone. Ironically, the root of the manifestation of $C$. difficile infections is, in most cases, also the treatment and as such there is not only an ongoing need for antibiotics with selectivity against $C$. difficle but also an ongoing challenge of preventing the recurrence of infection after antibiotic treatment, which occurs in a quarter of patients. Other reasons behind the development of $C$. difficile colitis include individuals with a weakened immune system or those undergoing chemotherapy where the host defences are compromised. Patients with a recurring infection have a $25 \%$ chance of being treated with antibiotics with $7 \%$ of diagnosed patients succumbing to the infection within 30 days.

We have recently reported the discovery of a new class of antibiotics targeting the novel mechanosensitive channel of large conductance $(\mathrm{MscL})^{3-5}$ and demonstrated the effectiveness of one such antibiotic in treating a methicillin-resistant Staphylococcus aureus infection in the model nematode Caenorhabditis elegans. ${ }^{4} \mathrm{MscL}$ is a much-desired antibiotic target owing to its high protein conservation across bacterial species and its absence in the human genome. MscL rescues bacteria from lysis by responding to the high turgor pressure within when bacteria are exposed to a high osmotic environment. As they open up, MscL channels allow the release of solutes and small proteins and alleviate the tension across the cell membrane. We have found that this new class of antibiotics lower the threshold at which the channel opens and extends its opening times, thereby slowing the growth of bacteria. ${ }^{4}$

Ramizol, indicated for the treatment of $C$. difficile colitis infections, was found to be superior to the commercially available vancomycin in exhibiting an in vitro concentration-dependent efficacy against

\footnotetext{
${ }^{1}$ School of Pharmacy and Medical Science, University of South Australia, Adelaide, SA, Australia; ${ }^{2}$ Eurofins Panlabs Ltd, Taipei, Taiwan; ${ }^{3}$ Micromyx LLC., Kalamazoo, MI, USA; ${ }^{4}$ School of Chemical and Physical Sciences, Flinders University, Bedford Park, SA, Australia and ${ }^{5}$ Antibiotic Development, Boulos \& Cooper Pharmaceuticals Pty Ltd, Port Adelaide, SA, Australia

Correspondence: Dr RA Boulos, Antibiotic Development, Boulos \& Cooper Pharmaceuticals Pty Ltd, 6 Todd Street, Port Adelaide, SA 5015, Australia.

E-mail: CEO@bouloscooper.com

Received 17 December 2015; revised 1 March 2016; accepted 13 March 2016; published online 18 May 2016
} 
S. aureus. ${ }^{4,6}$ However, it is poorly soluble in water (without the addition of base) and previous studies were conducted utilizing DMSO to solubilize the antibiotic. The use of DMSO as a solvent is tolerated at low concentrations in establishing proof-of-concept models for the efficacy of drugs in vitro; however, its use beyond preclinical work may introduce safety issues. Hence the use of a benign and efficacious aqueous drug formulation is desirable for clinical trials and for the successful development of drugs.

Here, we investigate the preclinical development of Ramizol utilizing a polysorbate 80 surfactant-based systems for the delivery of the novel compound. Impact of the delivery systems on the compound solubility in aqueous medium was studied using dynamic light scattering analysis. The potential of the FDA approved polysorbate 80 solubiliser to overcome the solubility issues of Ramizol was confirmed in an in vivo pharmacokinetic studies in an Sprague-Dawley rat model. In addition, in vitro efficacy against C. difficile as well as the in vivo efficacy studies in a hamster C. difficile colitis model were conducted to illustrate the effectiveness of the Ramizol delivery system. Lastly, we investigate the frequency of spontaneous mutants arising due to the use of Ramizol, to predict the long-term success of the treatment in a clinical setting.

\section{RESULTS}

Solubility and stability of Ramizol

Accelerated stability testing using HPLC of the Ramizol dry powder has shown no significant changes in peak area during the entire study period of 3 months, suggesting the dry powder is chemically stable (Supplementary Table S1). The droplet size of Ramizol in polysorbate 80 (results are expressed as the mean \pm s.d. of three replicates) suggest the use of polysorbate 80 was significantly more effective at reducing the size of the particles compared with the control (Table 1). Accelerated stability testing using HPLC of Ramizol in polysorbate 80 showed one peak present during the entire study period of 3 months, suggesting no chemical degradation (Supplementary Figure S1).

\section{In vitro activity of Ramizol}

Ramizol dissolved and diluted in DMSO had an in vitro antibacterial activity against a number $C$. difficile strains of different ribotypes in Reinforced Clostridial broth (MIC 4-8 $\mu \mathrm{g} \mathrm{ml}^{-1}$ ) and Reinforced Clostridial agar (MIC 16-32 $\mu \mathrm{g} \mathrm{ml}^{-1}$ ) (Table 2), with the four-fold increase in MIC in agar not being uncommon. ${ }^{7}$ For comparison, the MICs of fidaxomicin, metronidazole, moxifloxacin, rifaximin and vancomycin against $C$. difficile ribotype 027 (strain ATCC BAA-1805) were $0.0078,0.126,32,<0.0078$ and $0.6 \mu \mathrm{g} \mathrm{ml}^{-1}$, respectively.

Consistent with the reduction of the particle sizes and higher solubility, the MIC of Ramizol in polysorbate 80 against $C$. difficile strains were lower $(8 \times$ in the case of $C$. difficile, Ribotype 078)

Table 1 Particle size analysis of Ramizol in DMSO and in polysorbate 80 using DLS

\begin{tabular}{lcc}
\hline Additive & Solvent & Mean diameter $(\mathrm{nm}) \pm$ s.d. \\
\hline $1 \%$ DMSO & PBS & 1332 \\
$100 \%$ DMSO & - & $476.6 \pm 104.3$ \\
$2 \%$ polysorbate 80 & PBS & $439.53 \pm 12.12$ \\
$10 \%$ polysorbate 80 & PBS & $223.87 \pm 87.02$ \\
\hline
\end{tabular}

Abbreviations: DLS, dynamic light scattering; DMSO, dimethyl sulfoxide; PBS, phosphatebuffered saline. than those of the drug dissolved in DMSO (Supplementary Table S2).

\section{Interaction of Ramizol with other ion channels}

The radio-ligand binding assays of calcium, potassium and sodium ion channels showed no significant activation or inhibition when expressing cells were exposed to $10 \mu \mathrm{M}$ Ramizol (Supplementary Table S3).

\section{In vivo pharmacokinetics}

The plasma profiles of drug in polysorbate 80 were characterized with very little inter-variability. The mean dose-normalized plasma PK profiles show a maximum plasma concentration, $C_{\max }$, of $410.80 \mu \mathrm{g} \mathrm{ml}^{-1}$ when Ramizol was administered intravenously (at $5 \mathrm{mg} \mathrm{kg}^{-1}$ ) and the area under the curve was 653.36 and $692.15 \mu \mathrm{g} . \mathrm{h} \mathrm{ml}^{-1}$ for $\mathrm{AUC}_{0-24 \mathrm{~h}}$ and $\mathrm{AUC}_{0-\infty}$, respectively (Table 3). When administered orally at $5 \mathrm{mg} \mathrm{kg}^{-1}$, Ramizol had a maximum plasma concentration, $C_{\max }$, of $0.16 \mu \mathrm{g} \mathrm{ml}^{-1}$ and the area under the curve (AUC) was 0.91 and $1.38 \mu \mathrm{g} . \mathrm{h} \mathrm{ml}^{-1}$ for $\mathrm{AUC}_{0-24 \mathrm{~h}}$ and $\mathrm{AUC}_{0-\infty}$, respectively (Table 3 ). The absolute bioavailability (F) was calculated by dividing the $\mathrm{AUC}_{0-24 \mathrm{~h}}$ following the oral administration of Ramizol in polysorbate 80 by the $\mathrm{AUC}_{0-24 \mathrm{~h}}$ after the intravenous administration of the Ramizol in polysorbate 80 . The absolute bioavailability of Ramizol was extremely low with $<0.1 \%$ being absorbed (Figure 1).

\section{In vivo efficacy of Ramizol}

The efficacy of Ramizol was evaluated in the C. difficile colitis model using Golden Syrian hamsters and strain ATCC BAA-1805, a ribotype 027 NAP-1 epidemic strain. Oral administration of Ramizol at $100 \mathrm{mg} \mathrm{kg}^{-1}$ twice daily (b.i.d.) for 5 consecutive days was associated with a significant increase (57\%) in the survival rate of hamsters during the 28- day observation period (Figure 2). At the $50 \mathrm{mg} \mathrm{kg}^{-1}$ Ramizol dose, $43 \%$ of the hamsters survived while in the vehicle control group, all hamsters succumbed to infection by day 3 after infection. The reference substance, vancomycin at $20 \mathrm{mg} \mathrm{kg}^{-1}$ dosed orally b.i. d. for 5 consecutive days also elicited a significant increase in the survival rate $(86 \%)$ compared with the vehicle. There was a $2-\log$ decrease in spore count for all treatment groups with vancomycin, Ramizol at $50 \mathrm{mg} \mathrm{kg}^{-1}$ and Ramizol at $100 \mathrm{mg} \mathrm{kg}^{-1}$ showing $<1.53 \log 10,1.57 \log 10$ and $1.83 \log 10$ spore counts, respectively, compared with the vehicle group showing $3.78 \log 10$ spore counts per gram stool. Twenty-nine percent and $57 \%$ of animals in the group administered $50 \mathrm{mg} \mathrm{kg}^{-1}$ and $100 \mathrm{mg} \mathrm{kg}^{-1}$ Ramizol, respectively, had no detectable spores while $100 \%$ of animals in the vancomycin group (20 $\mathrm{mg} \mathrm{kg}^{-1}$ ) showed spore clearance below the level of detection. It is worth noting that none of the hamsters administered Ramizol suffered any diarrhetic side effects possibly suggesting little or no disruption to the gut flora.

Table 2 In vitro activity of Ramizol in Reinforced Clostridial agar and Reinforced Clostridial broth

\begin{tabular}{lcc}
\hline Strain & MIC $\left(\mu \mathrm{g}^{-1}\right)$ in broth & $M I C\left(\mu \mathrm{ml}^{-1}\right)$ in agar \\
\hline C. difficile ATCC 700057 & 4 & 16 \\
C. difficile ATCC BAA-1805 & 4 & 32 \\
C. difficile ATCC 43596 & 8 & 32 \\
C. difficile ATCC 43255 & 4 & 16
\end{tabular}


Table 3 Comparison of mean pharmacokinetic parameters after intravenous and oral administration of Ramizol dosed in polysorbate 80 at $5 \mathrm{mg} \mathrm{kg}^{-1}$

\begin{tabular}{lcc}
\hline & Intravenous & Oral \\
\hline$T_{\max }(\mathrm{h})$ & $0.25 \pm 0.00$ & $1.10 \pm 0.24$ \\
$T_{1 / 2}(\mathrm{~h})$ & $8.49 \pm 0.62$ & $10.50 \pm 3.92$ \\
$C_{\max }\left(\mu \mathrm{g} \mathrm{ml}{ }^{-1}\right)$ & $410.80 \pm 8.47$ & $0.16 \pm 20.04$ \\
$\mathrm{AUC}_{0-24 \mathrm{~h}}\left(\mu \mathrm{g} \cdot \mathrm{h} \mathrm{ml}^{-1}\right)$ & $653.36 \pm 29.14$ & $0.91 \pm 119.72$ \\
$\mathrm{AUC}_{0-\infty}\left(\mu \mathrm{g} \cdot \mathrm{h} \mathrm{ml}^{-1}\right)$ & $692.15 \pm 26.15$ & $1.38 \pm 250.79$ \\
$\mathrm{~F}$ & - & $0.09 \%$ \\
\hline
\end{tabular}

Data expressed as mean \pm s.e.m., $n=4$

\section{Spontaneous mutation frequency}

The frequency in which $C$. difficile strains spontaneously develop resistance to Ramizol was assessed by applying cultures of strains ATCC 700057 and ATCC 43255 to agar medium containing Ramizol at concentrations four- and eightfold the MIC on Clostridial Agar medium $\left(16 \mu \mathrm{g} \mathrm{ml}^{-1}\right)$ (Table 4). Despite inoculating the Ramizol containing plates with a very high C. difficile inoculum, no spontaneous resistant mutants grew at either $4 \times$ or $8 \times$ the MIC for Ramizol. The calculated spontaneous resistance mutation frequency from this study is very low, $<7.69 \times 10^{-13}$ for $C$. difficile ATCC 700057 and $<1.0 \times 10^{-12}$ for ATCC 43255. It is worth noting that the comparator antibiotic fidaxomicin also showed no spontaneous resistant mutants growing in this study.

\section{DISCUSSION}

Polysorbates and other amphiphilic excipients have been extensively used in pharmaceutical formulations preparation owing to their prowess in solubilizing sparingly soluble drugs. Their efficacy stems from the presence of hydrophilic and hydrophobic moieties within the same molecule acting in essence as a surfactant. Dynamic light scattering measurements have shown the use of polysorbate 80 was successful in solubilizing Ramizol and reducing its particle size to submicron dimensions. In contrast, control samples prepared in the presence of DMSO (1\%) have shown Ramizol particles greater than $5 \times$ in size compared with those in polysorbate 80 . The increased particle sizes are a direct result of aggregation because of the planar nature of the drug, enhanced by the presence of aromatic rings capable of inter-molecular $\pi-\pi$ stacking. ${ }^{8}$ Consistent with the improved solubility, a comparison of susceptibility testing against $C$. difficile strains has shown a reduction in MICs (Supplementary Table S2). This suggests that solubility is a key factor for improving the efficacy of Ramizol.

Drug stability assessment is an important aspect of drug development with direct relevance to storage conditions, shelf life and possibly market penetration. An example of this comes from the need of room temperature storage conditions $\left(25^{\circ} \mathrm{C}\right)$ to prevent the decomposition of doxycycline, conditions scarcely available in sub-Saharan Africa, hardest hit by bacterial infection and where power and refrigeration are beyond the reach of most communities. We have shown that Ramizol was stable up to 3 months at room temperature and at $4{ }^{\circ} \mathrm{C}$ in the powder form and in polysorbate 80 with no decomposition side products as determined by HPLC (Supplementary Table S1 and Supplementary Figure S1). In addition, the stability of Ramizol was tested at $40{ }^{\circ} \mathrm{C}$ and a relative humidity of $75 \%$ with no drug degradation observed (results not shown).

No significant interactions between Ramizol and the physiologically very relevant calcium, potassium and sodium ion channels were

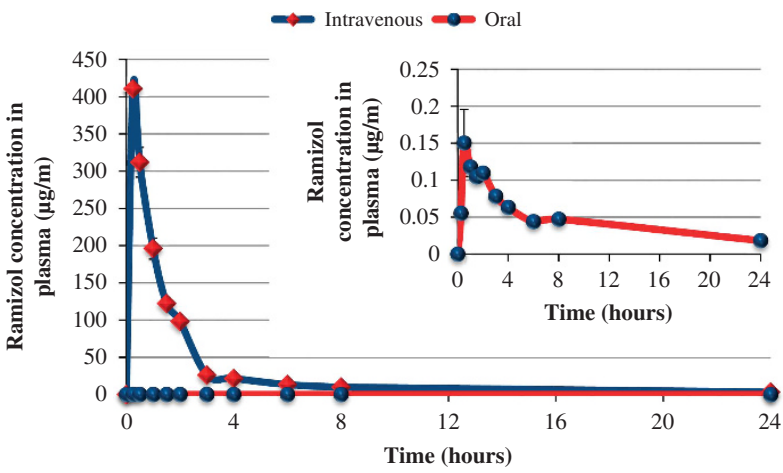

Figure 1 Mean dose-normalized plasma profiles after intravenous administration of $5 \mathrm{mg} \mathrm{kg}^{-1}$ Ramizol prepared in polysorbate 80 (Group 1). The inlay shows the mean dose-normalized plasma profiles after oral administration of $5 \mathrm{mg} \mathrm{kg}^{-1}$ Ramizol in polysorbate 80 (Group 2). Data is presented as mean \pm s.e.m. $(n=4)$.

observed. Calcium channel L-type has a role in cardiovascular disease, while the N-type mediate calcium influx in cells and directly affect synaptic transmission. The potassium channel $\left[\mathrm{K}_{\mathrm{ATP}}\right]$ has a role in modulating blood pressure, while the hERG type and the sodium channel are associated with cardiac arrhythmias. Non-specific interactions with these channels and especially the hERG, have presented roadblocks for a number of drugs in development ${ }^{9,10}$ including the antihistamine terfenadine and the antipsychotic droperidol which have been withdrawn from the market, ${ }^{10}$ and are the reason behind the cardiotoxicity of marcrolides, ketolides and fluoroquinolones. ${ }^{11}$ The lack of activity observed herein adds further credibility to the selectivity of this new class for $\mathrm{MscL}^{2}$ reducing the potential of unwarranted side effects.

We have found that when Ramizol was dosed intravenously in polysorbate 80 , it had a drug half-life of $8.49 \mathrm{~h}$ and a high $C_{\max }$ of $410.79 \mu \mathrm{g} \mathrm{ml}^{-1}$, suggesting rapid drug distribution. A plot of Log (plasma concentrations) versus time revealed a linear relationship (data not shown) for Ramizol suggesting the drug follows a single-compartment PK model, like aminoglycosides and unlike vancomycin. The extremely low bioavailability following oral administration suggests that Ramizol remained entirely in the gastrointestinal tract akin other non-systemic antibiotics such as vancomycin and fidaxomicin. This is not a surprising result given the presence of carboxylic groups which become deprotonated $\left(\mathrm{pK}_{\mathrm{a}} \sim 4.5\right)$ under the slightly basic $\mathrm{pH}$ environment in the large intestine, thus rendering the drug impermeable. These results classify Ramizol as a Class IV drug where it exhibits both poor solubility and poor gastrointestinal permeability.

We have found that Ramizol dosed at $50 \mathrm{mg} \mathrm{kg}^{-1}$ orally twice a day, saved $43 \%$ of the hamsters and when dosed at $100 \mathrm{mg} \mathrm{kg}^{-1}$ saved $57 \%$ of the hamsters, a statistically significant result. In comparison, vancomycin dosed at $20 \mathrm{mg} \mathrm{kg}^{-1}$ orally twice a day rescued $86 \%$ of the hamsters from infection. However it is worth noting that high-dose vancomycin does not prevent the recurrence of $C$. difficile infections in $20 \%$ of human subjects, ${ }^{12}$ highlighting the need for new therapeutic drugs. By increasing the frequency of doses from twice a day to four times a day, $71 \%$ of hamsters treated with a $100 \mathrm{mg} \mathrm{kg}^{-1}$ dose of Ramizol survived, while at $20 \mathrm{mg} \mathrm{kg}^{-1}$ Qid, $29 \%$ of the hamsters survived. Not surprising, and consistent with the oral pharmacokinetic data for Ramizol, the non-systemic nature of the drug is ideal for treating $C$. difficile colitis, whereby the drug is present in sufficient concentration to yield a therapeutic effect. The high dose required in the current study to observe a 


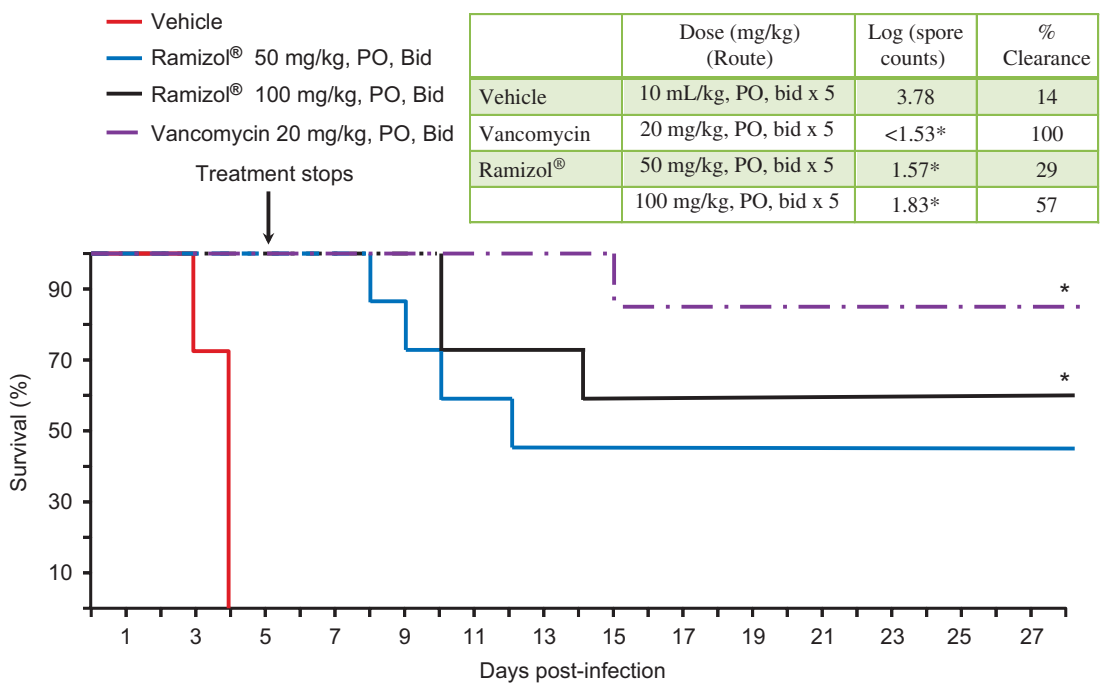

Figure 2 Effect of oral administration of Ramizol on survival rate in the hamster C. difficile (strain BAA-1805, 027 ribotype) -induced colitis. The animals were orally inoculated with $C$. difficile (BAA- 1805) at a lethal LD90-100 inoculum size $\left(1.35 \times 10^{5}\right.$ spores per animal) in $0.5 \mathrm{ml}$ PBS. Test substance was orally administered twice daily for 5 days starting $16 \mathrm{~h}$ after infection. Mortality was monitored for 28 days for all groups. The Fisher's exact test was used to determine the statistical significance compared with the untreated vehicle group at ${ }^{*} P<0.05$. Inset table shows the mean log (spore counts) in hamsters $(n=7)$ at the conclusion of the study for each study group. Effect of oral administration of Ramizol and vancomycin on fecal spore counts on day 3 in the hamsters infected with $C$. difficile strain BAA-1805, an 027 ribotype strain. The animals were orally inoculated with C. difficile (BAA-1805) at an LD90-100 inoculum size $(1.43 \times 105$ spores per animal) in $0.5 \mathrm{ml}$ PBS. Test substance was orally administered twice daily for 5 days starting $16 \mathrm{~h}$ after infection. The feces were collected on day 3 (40-64 h post infection) from surviving animals. A 2-log reduction (or more) in the spore counts of the treatment groups compared with the vehicle group indicated significant activity presented as $\left(^{*}\right)$. Fecal spore counts below the limit of detection (1.54) were indicated by \% clearance. Vehicle is $2 \%$ polysorbate $80 / 0.9 \% \mathrm{NaCl}$. LOD, limit of detection.

Table 4 Spontaneous mutation frequency of Ramizol for $C$. difficile isolates in agar

\begin{tabular}{|c|c|c|c|c|c|}
\hline Strain & $M I C$ & MIC multiple for mutant selection & Mean inoculum size (CFU $\mathrm{ml}^{-1}$ ) & Colony counts & Mutation frequency \\
\hline & & $4 \times$ & & 0 & \\
\hline & & $4 \times$ & & 0 & \\
\hline
\end{tabular}

therapeutic effect is a direct result of the poor solubility of Ramizol as a considerable proportion of the administered dose may have precipitated in the gastrointestinal fluids as polysorbate is diluted. This could be addressed by exploiting the presence of carboxylic acid moieties in the drug molecule for salt formation, an approach that will significantly improve the solubility of the drug in an aqueous medium. This coupled with an acid-resistant drug-loading capsule will allow for targeted drug delivery to the large intestine, provide for prolonged period of drug release while preventing drug precipitation at the same time, would offer great potential in significantly reducing the dose without compromising the efficacy.

The absence of spontaneous mutants in the presence of $4 \times$ or $8 \times$ the MIC of Ramizol, despite using very high inocula of two C. difficile isolates is clinically significant. The data suggest that C. difficile has a low chance of developing resistance to the antibiotic in the clinic and the drug is suitable as a monotherapy for the treatment of $C$. difficile associated disease.

In conclusion, we have presented the preclinical development of Ramizol, a first generation belonging to an entirely new class and have shown its effectiveness in treating $C$. difficile colitis in a hamster model. This new class targets the novel mechanosensitive ion channel of large conductance, exhibits low cytotoxicity, antioxidant properties, ease of chemical synthesis, scalability and low cost of goods, chemically stable nature and in the case of Ramizol, non-systemic exposure, all contributing factors to the competitive advantage of this antibiotic for treating $C$. difficile infections. Future work will include the development of a more efficient formulation and animal toxicity investigation before first-in-man clinical trials.

\section{MATERIALS AND METHODS}

\section{Chemicals}

Bacto agar (DIFCO from Becton Dickinson, Sparks, MD, USA), Supplemented Brucella Agar (Remel, Lenexa, KS, USA), Reinforced Clostridial Broth (HiMedia, Lot No. 0000186925, Mumbai, India), anaerobic blood agar plate (Cat\# A01-05, CMP, Taiwan), cycloserine cefoxitin fructose agar with horse blood and taurocholate (Cat\# A01-269, CMP, Taiwan), phosphate-buffered saline (PBS, pH 7.0) (in-house), sodium chloride (Cat\# S7653, Sigma, St Louis, MO, USA), water for injection (Tai-Yu, Taichung City, Taiwan), vancomycin (Cat\# V2002, Sigma), clindamycin 2-phosphate (Cat\# PHR1021, Sigma), polysorbate 80 (Chem-Supply, Gillman, SA, Australia).

Ramizol (purity $>98 \%$ by HPLC) was supplied by Boulos \& Cooper Pharmaceuticals (Port Adelaide, South Australia, Australia) and stored at $4{ }^{\circ} \mathrm{C}$ until assayed. The solvent for the preparation of the Ramizol stock solutions was $100 \%$ DMSO, prepared at a concentration of $1280 \mu \mathrm{g} \mathrm{ml}^{-1}$ for the MIC testing in Clostridial Broth and Agar, and $5120 \mu \mathrm{g} \mathrm{ml}^{-1}$ for the evaluation of resistance development. The medium employed for the agar dilution MIC assay was Reinforced Clostridial Broth with 1.5\% agar. The medium employed for the broth MIC assay was Reinforced Clostridial Broth. 
Reinforced Clostridial Broth (HiMedia, Lot No. 0000186925) supplemented with 1.5\% agar (Becton Dickinson, Lot No. 6310397) was used for the spontaneous mutation frequency assay.

\section{Organisms}

All test organisms for the assays were acquired from the American Type Culture Collection (ATCC, Rockville, MD, USA). The growth and test media were those recommended by Clinical \& Laboratory Standards Institute $(\mathrm{CLSI})^{13}$ for growth and susceptibility testing of anaerobes. The test organisms were frozen at $-80^{\circ} \mathrm{C}$ until needed. The isolates were sub-cultured on Supplemented Brucella Agar plates and incubated for $48 \mathrm{~h}$ at $35^{\circ} \mathrm{C}$ in the anaerobic environment (5\% hydrogen, 5\% carbon dioxide, $90 \%$ nitrogen) using a Bactron II anaerobic chamber (Sheldon Manufacturing Inc, Cornelius, OR, USA). C. difficile 4381 (ATCC 700057) and C. difficile 4994 (ATCC 43255) were studied in the spontaneous mutation frequency assay.

\section{Stability testing}

Ramizol (dry powder) was packed in aluminum foil and stored at room temp or $4{ }^{\circ} \mathrm{C}$ for up to 3 months. To test the drug stability, a weighed amount of drug dry powder was collected from each package on day 0 , week 1 , month 1 and month 3; the dry powder was dissolved in DMSO and diluted with HPLC mobile phase to obtain a drug solution of $1 \mu \mathrm{g} \mathrm{ml}^{-1}$ and the drug solution was analyzed using HPLC. Significant changes in the HPLC peak area were considered to reflect the chemical decomposition of the drug molecule. The data for week 1, month 1 and month 3 were presented as a \% change of peak area compared with the peak area at day 0 .

\section{Preparation of Ramizol in polysorbate 80 and characterization}

Ramizol was prepared in polysorbate 80 in PBS at a concentration of approximately $2 \mathrm{mg} \mathrm{ml}^{-1}$. Ramizol in polysorbate 80 was diluted 100 times with water before the analysis of droplet size via phase analysis light scattering (Malvern Zetasizer Nano ZS, Malvern, UK). As a control, Ramizol was also prepared in $100 \%$ in DMSO, and in 100\% DMSO diluted 100 times with PBS to produce a $1 \%$ final DMSO concentration. Drug loading levels of the drug in polysorbate 80 was determined by first diluting the sample 100 times with the HPLC mobile phase (Acetonitrile and $5 \mathrm{~mm}$ phosphoric acid, $\mathrm{pH}=3,55: 45, \mathrm{v} / \mathrm{v}$ ) and analyzed for the drug content by HPLC. Studies were conducted in triplicate, and drug-loading levels were expressed as mean \pm s.d.

\section{Radioligand binding assays of mammalian channels}

The assays were conducted following previously published literature protocols. ${ }^{14-19}$

\section{Pharmacokinetic investigation in a rat model}

The animal experiments were approved by the Animal Ethics Committee, University of South Australia (Australia). Healthy male Sprague-Dawley rats weighing from 220 to $320 \mathrm{~g}$ were used for the PK dose study. Before drug dosing, each rat was cannulated in the right jugular vein under inhaled isoflurane and allowed to recover for 1 day. The rats were divided into 2 treatment groups $(n=4)$ : Group 1 -Ramizol $\left(5 \mathrm{mg} \mathrm{kg}^{-1}\right)$ prepared in polysorbate $80\left(5 \mathrm{mg} \mathrm{kg}^{-1}\right)$ administered intravenously; and Group 2-Ramizol in polysorbate 80 administered orally $\left(5 \mathrm{mg} \mathrm{kg}^{-1}\right)$. Food and water was accessible to all rats in Group 1 throughout the study. The rats in Group 2 were fasted $8 \mathrm{~h}$ before the dosage and $2 \mathrm{~h}$ post the oral dosage but had access to food and water at other times.

Plasma samples underwent a protein precipitation procedure before analysis on the HPLC system. Briefly, $50 \mu \mathrm{l}$ plasma was mixed with $2 \mu \mathrm{l}$ phosphoric acid $(\mathrm{pH} 3)$ and $48 \mu \mathrm{l}$ acetonitrile, sonicated for $5 \mathrm{~min}$ and centrifuged at 20000 r.p.m. for $5 \mathrm{~min}$. The supernantant was diluted properly with the mobile phase before HPLC analysis. Plasma standards were also prepared in a similar manner with the addition of drug stock solutions to provide a standard reference range of $50-1000 \mathrm{ng} \mathrm{ml}^{-1}$ in plasma. Plasma concentrations were dose-normalized to $5 \mathrm{mg} \mathrm{kg}^{-1}$ to account for variation in animal weight and plotted as plasma drug concentration $\left(\mu \mathrm{g} \mathrm{ml}^{-1}\right)$ versus time $(\mathrm{h})$

\section{C. difficile colitis hamster infection model}

The hamster infection model was similar to that published previously ${ }^{20}$ but performed with $C$. difficile strain BAA-1805, from the ATCC. ${ }^{21}$ The strain was cultured on anaerobic blood agar plates and incubated under anaerobic conditions $(80 \%$ nitrogen, $10 \%$ carbon dioxide and $10 \%$ hydrogen) $35-37^{\circ} \mathrm{C}$ for 5 days. The growth on the plates was transferred to PBS and heated at $70{ }^{\circ} \mathrm{C}$ for $30 \mathrm{~min}$ to inactivate the vegetative cells. Spores were pelleted by centrifugation at $3300 \mathrm{~g}$ for $15 \mathrm{~min}$ and resuspended in cold PBS then diluted in PBS to $2.7 \times 10^{5}$ spores per $\mathrm{ml}$ as determined with dilution plating.

Male golden Syrian hamsters weighing $90 \pm 10 \mathrm{~g}$ were provided by the National Laboratory Animal Center, Taiwan. The animals were individually housed in individually ventilated cages $(30 \times 19 \times 13 \mathrm{~cm}$ in size), with controlled temperature $\left(20-24^{\circ} \mathrm{C}\right)$, humidity $(30-70 \%)$ and $12 \mathrm{~h}$ light/dark cycles. Free access to standard lab diet (MFG (Oriental Yeast Co., Ltd., Tokyo, Japan)) and autoclaved tap water were granted. All aspects of this work including housing, experimentation, and animal disposal were performed in general accordance with the Guide for the Care and Use of Laboratory Animals: Eighth Edition (National Academies Press, Washington, DC, 2011) in the AAALAC-accredited ABSL2 laboratory animal facility. In addition, the animal care and use protocol was reviewed and approved by the IACUC at Eurofins Panlabs Taiwan, Ltd, Taipei, Taiwan).

Hamsters were treated with a single subcutaneous administration of clindamycin at $50 \mathrm{mg} \mathrm{kg}^{-1} 1$ day before infection $($ day -1$)$ to render animals susceptible to $C$. difficile infection. On day 0 , animals were orally inoculated with $1.4 \times 10^{5}$ spores in $0.5 \mathrm{ml}$ PBS. Ramizol, dissolved in $2 \%$ polysorbate $80 / 0.9 \% \mathrm{NaCl}$, was administered to animal groups at 50 and $100 \mathrm{mg} \mathrm{kg}^{-1}$ twice daily for 5 consecutive days starting $16 \mathrm{~h}$ after spore inoculation. Vancomycin, dissolved in $0.9 \% \mathrm{NaCl}$, was orally administered at $20 \mathrm{mg} \mathrm{kg}^{-1}$ twice daily for 5 days as the QC standard. Mortality was observed for 28 days. The Fisher's exact test was used to ascertain the statistical significance of animal survival compared with vehicle group with a significance criterion of $P<0.05$. To determine fecal spore counts, stools were collected from animals at $40-64 \mathrm{~h}$ after infection. Samples were homogenized in PBS, heated $30 \mathrm{~min}$ at $80^{\circ} \mathrm{C}$ to kill vegetative cells, then dilutions were plated onto cycloserine cefoxitin fructose agar with horse blood and taurocholate plates for colony counts.

\section{Hamster infection}

Groups of five or seven male golden Syrian hamsters weighing $90 \pm 10 \mathrm{~g}$ were used. Animals were pretreated with a single subcutaneous administration of clindamycin at $50 \mathrm{mg} \mathrm{kg}^{-1} 1$ day before infection (day -1). On day 0 , animals were orally inoculated $(0.5 \mathrm{ml}$ per animal $)$ with the targeted inoculum size at $1.0 \times 10^{5}$ spores per animal (the actual inoculum sizes were $1.35 \times 10^{5}$ spores per animal) of $C$. difficile (BAA-1805). Test substance, Ramizol, was administered at 50 and $100 \mathrm{mg} \mathrm{kg}^{-1}$ two times per day for 5 days starting $16 \mathrm{~h}$ after inoculation. An additional group for testing on vancomycin administered at $20 \mathrm{mg} \mathrm{kg}^{-1}$ twice daily (bid, PO) for 5 days was the QC standard. Mortality was observed for 28 days. An increase of 50 percent or more in survival rate compared with the negative control group indicated significant anti-infective activity. The Fisher's exact test was also used to ascertain the statistical significance compared with vehicle group at $P<0.05$.

\section{MIC assay-broth microdilution method}

MIC values were determined using a broth microdilution procedure described by the CLSI. ${ }^{13}$ Automated liquid handlers (Multidrop 384, Labsystems, Helsinki, Finland; Biomek 2000 and Biomek FX, Beckman Coulter, Fullerton, CA, USA) were used to conduct serial dilutions and liquid transfers. Test articles were dissolved in $100 \%$ vehicle or and diluted by two-fold serial titrations in 96-well microdilution plates (Costar 3795, Corning, NY, USA). Test article solutions were further diluted 40-fold into Reinforced Clostridial Broth medium in wells of 96-well plates then incubated in a Bactron II Anaerobic Chamber to reduce for $\sim 1-2$ h. C. difficile inoculum was added to a final cell count of $1 \times 10^{6} \mathrm{CFU} \mathrm{ml}^{-1}$. The final diluent concentration was $2.5 \%$. Plates were incubated at $35^{\circ} \mathrm{C}$ for $\sim 48 \mathrm{~h}$ in BD Gaspak EZ Anaerobe containers (Becton Dickinson). Plates were visually inspected for growth and the MIC was recorded as the lowest concentration of test article that inhibited visible growth of the organism. 


\section{MIC assay-agar dilution method}

MIC values for anaerobic bacteria were determined using the agar dilution method of the CLSI. Organisms were grown in a Bactron II Anaerobic Chamber for approximately $48 \mathrm{~h}$ before assay. Drug dilutions and drug-supplemented agar plates were prepared manually. The plates were allowed to stand at room temperature for $1-2 \mathrm{~h}$ to allow the agar surface to dry, reduced for $\sim 1-2 \mathrm{~h}$, and then spot-inoculated using a Steers Replicator (Melrose Machine Shop, Woodlyn, PA, USA). Spot-inoculation yielded a final cell concentration on the surface of the agar plates of $\sim 1 \times 10^{5}$ colony forming-units/spot. After the inocula had dried, the drug-supplemented plates were incubated at $35^{\circ} \mathrm{C}$ for approximately $48 \mathrm{~h}$ in BD Gaspak EZ Anaerobe containers (Becton Dickinson). After incubation, plates were removed from the containers and the MICs were read per CLSI guidelines.

\section{Measurement of the spontaneous mutation frequency}

The spontaneous mutation frequency method followed standard procedures published previously. ${ }^{22-24}$ C. difficile strains ATCC 700057 and ATCC 43255 were used for the resistance study. Plates containing Ramizol were prepared by diluting a stock solution 40-fold into molten clostridial agar medium to final concentrations of four- and eightfold the agar MIC, 64 and $128 \mu \mathrm{g} \mathrm{ml}^{-1}$, respectively. The drug/agar mixture was dispensed into sterile $15 \mathrm{~cm}$ plates (BD Falcon, Franklin Lakes, NJ, USA) to a volume of $50 \mathrm{ml}$ in each plate. Plates were allowed to solidify at room temperature and further dried in a SterilGARD II biological hood. Plates were then reduced for $\sim 1-2 \mathrm{~h}$ in the Bactron II Anaerobic Chamber. Bacterial growth from freshly streaked plates of each $C$. difficile test strain was harvested with sterile cotton swabs and suspended in $4 \mathrm{ml}$ of supplemented Brucella broth. This cell suspension constituted the inoculum for the spontaneous mutation study. The viable count of this suspension was determined by plating serial 10 -fold dilutions onto a drug-free Reinforced Clostridial agar with colony counting. For the spontaneous mutation assay, $0.1 \mathrm{ml}$ of inoculum was spread onto the surface of duplicate $15 \mathrm{~cm}$ plates containing Ramizol at 64 or $128 \mu \mathrm{g} \mathrm{ml}^{-1}$. The plates were incubated at $35^{\circ} \mathrm{C}$ for $48 \mathrm{~h}$ in the anaerobic environment (5\% hydrogen, 5\% carbon dioxide and $90 \%$ nitrogen) before colony counting.

The spontaneous mutation frequency was calculated using the following equation:

\section{Average number of colonies on Ramizol selection plate} Total number of cells inoculated onto the plate

If there were no colonies on a given antibiotic selection plate, the spontaneous mutation frequency was reported as less than or equal to the spontaneous mutation frequency that would have resulted from the presence of one colony (for example, one per total number of cells inoculated onto the plate).

\section{CONFLICT OF INTEREST}

RAB is the Chief Executive Officer of Boulos \& Cooper Pharmaceuticals Pty Ltd, which owns the intellectual property. The remaining authors declare no conflict of interest.

\section{ACKNOWLEDGEMENTS}

We gratefully acknowledge funding from Boulos \& Cooper Pharmaceuticals PTY Ltd for supporting the project. Ramizol is a trademark fully registered in Australia.

Author contributions: SR carried out the validation of the HPLC, the stability testing and the pharmacokinetic studies of Ramizol. DS and DLS carried out the spontaneous mutation frequency testing and the susceptibility testing in agar and $\mathrm{RAB}$ developed the polysorbate-containing preparations used in the studies. SR, CAP, LM, DS, DLS and RAB designed the experiments and RAB coordinated the research.
1 US Department of Health and Human Services Center for Disease Control and Prevention. Antibiotic Resistance Threats in the United States (2013). http://www. cdc.gov/drugresistance/threat-report-2013/index.html.

2 Butler, M. S., Blaskovich, M. A. \& Cooper, M. A. Antibiotics in the clinical pipeline in 2013. J. Antibiot. (Tokyo) 66, 571-591 (2013).

3 James, E. et al. A novel antimicrobial agent reduces oxidative stress in cells. RSC Adv. 3, 7277-7281 (2013).

4 Iscla, I. et al. A new antibiotic with potent activity targets MscL. J. Antibiot. (Tokyo) 68, 453-462 (2015).

5 Boulos, R. A. et al. Inspiration from old dyes: tris(stilbene) compounds as potent grampositive antibacterial agents. Chemistry 19, 17980-17988 (2013).

6 Rybak, M. J. The pharmacokinetic and pharmacodynamic properties of vancomycin. Clin. Infect. Dis. 42, S35-S39 (2006).

7 Waites, K. B. et al. Comparison of agar versus broth dilution techniques for determining antibiotic susceptibilities of Ureaplasma urealyticum. Diagn. Microbiol. Infect. Dis. 14, 265-271 (1991).

8 Wahid, M. H. et al. Aqueous based synthesis of antimicrobial-decorated graphene. J. Colloid Interface Sci. 443, 88-96 (2015).

9 Sanguinetti, M. C. \& Tristani-Firouzi, M. hERG potassium channels and cardiac arrhythmia. Nature 23, 463-469 (2006).

10 Priest, B. T., Bell, I. M. \& Garcia, M. Role of hERG potassium channel assays in drug development. Channels 2, 87-93 (2008).

11 Lannini, P. B. Cardiotoxicity of macrolides, ketolides and fluoroquinolones that prolong the QTc interval. Expert Opin. Drug Saf. 1, 121-128 (2002).

12 Surawicz, C. M. et al. The search for a better treatment for recurrent Clostridium difficile disease: use of high-dose vancomycin combined with Saccharomyces boulardii. Clin. Infect. Dis. 31, 1012-1017 (2000).

13 Clinical and Laboratory Standards Institute. Methods for Antimicrobial Susceptibility Testing of Anaerobic Bacteria; Approved Standard-Eighth Edition. CLSI document M11-A8 (CLSI, Wayne, PA, USA, 2012).

14 Schoemaker, H. \& Langer, S. Z. [3H]Diltiazem binding to calcium channel antagonists recognition sites in rat cerebral cortex. Eur. J. Pharmacol. 111, 273-277 (1985).

15 Ehlert, F. J., Roeske, W. R., Itoga, E. \& Yamamura, H. I. The binding of [3H] nitrendipine to receptors for calcium channel antagonists in the heart, cerebral cortex, and ileum of rats. Life Sci. 30, 3656-3660 (1982).

16 Moresco, R. M., Govoni, S., Battaini, F., Trivulzio, S. \& Trabucchi, M. Omegaconotoxin binding decreases in aged rat brain. Neurobiol. Aging 11, 433-436 (1990).

17 Gaines, K. L., Hamilton, S. \& Boyd, III A. E. Characterization of the sulfonylurea receptor on beta cell membranes. J. Biol. Chem. 263, 2589-2592 (1988).

18 Finlayson, K., Turnbull, L., January, C. T., Sharkey, J. \& Kelly, J. S. [3H]dofetilide binding to HERG transfected membranes: a potential high throughput preclinical screen. Eur. J. Pharmacol. 430, 147-148 (2001).

19 Catterall, W. A., Morrow, C. S., Daly, J. W. \& Brown, C. B. Binding of batrachotoxinin A 20-alpha-benzoate to a receptor site associated with sodium channels in synaptic nerve ending particles. J. Biol. Chem. 256, 8922-8927 (1981).

20 Ochsner, U. A. et al. Inhibitory effect of REP3123 on toxin and spore formation in Clostridium difficile, and in vivo efficacy in a hamster gastrointestinal infection model. J. Antimicrob. Chemother. 63, 964-971 (2009).

21 Clostridium difficile ribotype 027 (BAA-1805), Hamster Colitis Model. Available at: https://www.eurofinspanlabs.com/Catalog/Products/ProductDetails.aspx? prodld $=$ tkXBeXQXKFA\%3D.

22 Montgomery, A. B., Rhomberg, P. R., Abuan, T., Walters, K. A. \& Flamm, R. K. Amikacin-fosfomycin at a five-to-two ratio: characterization of mutation rates in microbial strains causing ventilator-associated pneumonia and interactions with commonly used antibiotics. Antimicrob. Agents Chemother. 58, 3708-3713 (2014).

23 Fung-Tomc, J., Huczko, E., Pearce, M. \& Kessler, R. E. Frequency of in vitro resistance of Pseudomonas aeruginosa to cefepime, ceftazidime, and cefotaxime. Antimicrob. Agents Chemother. 32, 1443-1445 (1988).

24 Kaatz, G. W. et al. Efficacy of trovafloxacin against experimental Staphylococcus aureus endocarditis. Antimicrob. Agents Chemother. 42, 254-256 (1998).

This work is licensed under a Creative Commons Attribution-NonCommercial-NoDerivs $\quad 4.0$ International License. The images or other third party material in this article are included in the article's Creative Commons license, unless indicated otherwise in the credit line; if the material is not included under the Creative Commons license, users will need to obtain permission from the license holder to reproduce the material. To view a copy of this license, visit http://creativecommons.org/licenses/bync-nd/4.0/

Supplementary Information accompanies the paper on The Journal of Antibiotics website (http://www.nature.com/ja) 